\title{
ORIGINAL
}

\section{Epileptic seizure discharges in patients after open chamber cardiac surgery-a prospective prevalence pilot study using continuous electroencephalography}

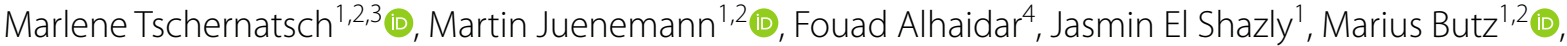 \\ Marco Meyer ${ }^{5}$, Tibo Gerriets ${ }^{1,2,3}$, Markus Schönburg ${ }^{1,6}$ (D) and Patrick Schramm ${ }^{1,7^{*}}$ (D)
}

(C) 2020 The Author(s)

\begin{abstract}
Purpose: Patients undergoing cardiac surgery often develop delirium which increases the risk of postoperative morbidity and leads to a reduced quality of life. Retrospective studies show a higher incidence of delirium in patients with seizures. However, these studies do not systematically detect subclinical seizures, so the incidence of seizures after cardiac surgery remains speculative. The objective of this study is to determine the prevalence of electrographic seizures after elective open-chamber cardiac surgery.

Methods: This prospective, blinded, monocentric, observational study investigated patients scheduled for elective open-chamber valve reconstruction or replacement. Anaesthesia, surgery and postoperative treatment were standardized and not influenced by the presented observation. After surgery, all patients arrived at the ICU, and EEG monitoring started within the first hour. EEG recording was continuously performed for up to $24 \mathrm{~h}$, and the results were independently analysed by two blinded EEG board-certified neurologists.

Results: 100 patients were included. Abnormal EEG patterns were present in 33\% of patients, and $9 \%$ of all patients showed electrographic seizures. The main EEG activity at the beginning of each recording was suppressed or showed a burst-suppression pattern, and at the end of recording, all patients had an alpha/theta rhythm. An association between electrographic seizures and delirium was found $\left(p_{x_{2}}<0.01\right)$.

Conclusion: This study reveals a surprisingly high incidence of abnormal EEG patterns and electrographic seizures in patients undergoing open-chamber cardiac surgery. As electrographic seizures are associated with the incidence of delirium, this finding is a relevant phenomenon in the post-cardiac surgery ICU population.
\end{abstract}

Keywords: Electroencephalography, Cardiac surgery, Seizures, Electrographic seizures, Delirium

\footnotetext{
*Correspondence: schrammp@uni-mainz.de

7 Department of Anaesthesiology, University Medical Center of the Johannes Gutenberg-University, Langenbeckstrasse 1, 55131 Mainz, Germany

Full author information is available at the end of the article

Marlene Tschernatsch and Martin Juenemann have contributed equally

to this work.
}

\section{晋




\section{Introduction}

Patients undergoing cardiac surgery often develop delirium and postoperative cognitive deficits (POCD), which leads to a higher risk of postoperative morbidity and mortality and a reduced quality of life [1]. Retrospective studies show a higher incidence of delirium, stroke and mortality in patients with convulsive and non-convulsive seizures after cardiac surgery [2,3]. However, these studies consider either clinically obvious, generalised, convulsive seizures or non-convulsive seizures, the latter of which are diagnosed by a corresponding pathological EEG examination, with the indication to perform such an examination remaining uncertain. Overall, these studies indicate an incidence of postoperative epileptic seizures of $1-2.5 \%$ but do not systematically investigate the incidence of subclinical seizures in the early postoperative period. Gofton et al. analysed an unselected cardiac surgery collective using a continuous subhairline EEG, finding seizures in 3\% of the patients, all of whom underwent open-chamber surgery [4]. However, the use of subhairline electrodes leads to underdiagnosis of seizures [5, 6]. In summary, the incidence of seizures after cardiac surgery remains speculative. Seizures are treatable and, therefore, represent a potential target in the prophylaxis of delirium and POCD.

To investigate the accurate prevalence of seizures, we conducted this prospective study on patients undergoing open-chamber cardiac surgery using continuous EEG monitoring with widely distributed electrodes in a 10-channel registration.

\section{Methods}

This prospective, blinded, monocentric, observational study was conducted at Kerckhoff-Hospital, Bad Nauheim, Germany, from January 2016 to December 2017. After gaining approval from the local ethical committee of Justus-Liebig-University Giessen, Germany, and obtaining written informed consent, consecutive patients scheduled for elective open-chamber aortic or mitral valve reconstruction or replacement were included. Exclusion criteria were emergency surgery, lack of informed consent, pregnancy, pre-existing epilepsy, surgery in deep hypothermia or off-pump surgery. Patients were screened for pre-existing cognitive deficits using the mini-mental status examination (MMSE) the day before surgery. The anaesthesia, surgery and postoperative treatment were standardised and not influenced by the presented observation. The anaesthesia induction included $2.5-3 \mathrm{mg} / \mathrm{kg}$ propofol and a $0.8-1 \mu \mathrm{g} / \mathrm{kg}$ sufentanil bolus. Propofol was applied continuously, and remifentanil was added at a rate of $0.15-1 \mu \mathrm{g} / \mathrm{kg}^{*} \mathrm{~min}$ for continuous analgesia. In addition to the continuous

\section{Take-home message}

This study reveals for the first time a surprisingly high incidence of 33\% abnormal EEG patterns, including 9\% that were electrographic seizures, after open-chamber cardiac valve surgery, which shows an association with the incidence of delirium.

remifentanil, sufentanil boli were applied before painful surgical procedures. The target of the bispectral index (BIS) was between 40 and 60 .

In order to prevent air embolism, $\mathrm{CO}_{2}$ was inflated into the open heart and air was manually squeezed out at the end of the bypass procedure. Patients were placed in a Trendelenburg position, and the aortic root vent evacuated the air outside of the cavum and the aorta. Transoesophageal echocardiography was used to confirm that no air remained, then the aortal clamp was opened and the patient was positioned horizontally. After surgery, all patients were admitted to the ICU with analgo-sedative drugs (remifentanil and propofol) and invasive ventilation. After the EEG monitoring was established, propofol and remifentanil were tapered with the goal of early extubation. Piritramide and metamizole were used to obtain analgesia.

EEG monitoring started within the first hour after the patients' admission to the ICU (EEG-1100-system, Nihon Kohden Europe $\mathrm{GmbH}$, Rosbach, Germany). An EEG was performed using 10 cup electrodes $(\mathrm{Ag} / \mathrm{AgCl})$ fixed at the scalp with electrode paste following abrasion of the skin surface. The electrodes were placed according to the 10-20 system at Fp1, Fp2, C3, C4, P3, P4, T3, T4, $\mathrm{Fz}$ and $\mathrm{Cz}$. EEG recording was performed continuously for up to $24 \mathrm{~h}$ unless patients were discharged from the ICU or more than three electrodes became dysfunctional. Afterwards, the EEG results were independently analysed with a focus on predominant background EEG frequencies and abnormal EEG patterns, such as periodic discharges, rhythmic delta activity, rhythmic or sporadic spikes, sharp-and-slow-waves, spike-and-wave and electrographic seizures, defined as patterns with evolution in amplitude and frequency, as determined by two EEG board-certified neurologists (MT, PS) [7, 8]. Both were blinded to patients' treatment and outcome parameters. The EEG interpretation was performed after patients' treatment, so the findings had no influence on the individual therapy. In the case of different interpretations, both investigators analysed the individual pattern together until a consensus was reached.

For secondary analysis, the incidence of delirium was assessed by nursing staff on the first and fourth postoperative day using the confusion assessment method for the intensive care unit (CAM-ICU). Furthermore, 
the patients' length of stay in the ICU and postoperative strokes up to the end of inpatient rehabilitation were recorded. Dosages of clorazepate for premedication, dosages of tranexamic acid, time of extracorporeal bypass, aortic clamping time, lowest temperature during cardiopulmonary bypass, intraoperative BIS and mean arterial blood pressure were recorded in the anaesthesia protocol and used to detect potentially uncontrollable influencing factors.

\section{Statistical analysis}

Due to the absence of preliminary data, a power analysis could not be performed. For this prevalence study, a sample size of 100 patients was aimed. The prevalence of seizures and epileptic discharges was analysed descriptively. For secondary analysis, a classification was performed of patients with/without abnormal EEG pattern and of patients with/without electrographic seizures. It was investigated whether the groups with/without abnormal EEG pattern and with/without electrographic seizures differed in patient characteristics (age, gender, BMI), preoperative mental state or intra-operative data. To verify whether the groups differed significantly in categorical variables, Chi-square tests were calculated on the basis of a dichotomous scale level of the variable. For parametric continuous variables, Student's $t$ tests were used. Continuous variables that did not meet the criteria of parametric testing were evaluated using Mann-Whitney $U$ test. Normal distribution was proven with the Shapiro-Wilk test and variance homogeneity with the Levene test. For all metric variables, mean values and standard deviation (SD) were reported; for categorically scaled parameters, frequency and percentage were given. Statistical analyses were calculated using SPSS (version 24, IBM ${ }^{\circledR}$ SPSS $^{\circledR}$ Statistics, USA). A significance level with $p<0.05$ was considered to be statistically significant.

\section{Results}

One hundred patients were included, $76 \%$ of whom underwent aortic valve replacement and $24 \%$ of whom endured mitral valve reconstruction or replacement. The mean age of the patients was $70 \pm 10$ years, $72 \%$ were male, and the mean BMI was $27 \pm 5 \mathrm{~kg} / \mathrm{m}^{2}$. The American Society of Anesthesiologists' physiological status classification was 3 [2; 4]. The mean MMSE before surgery was $28.3 \pm 1.7$. All patients who gave consent were measured, with no dropouts after the primary analysis. Clorazepate was used as premedication in $85 \%$ of the patients $(24 \pm 16 \mathrm{mg})$, and $99 \%$ of the patients received tranexamic acid $(2.8 \pm 0.7 \mathrm{~g}$, $\max 4.0 \mathrm{~g})$ during the surgery. Extracorporeal circulation was performed for $112 \pm 37 \mathrm{~min}$, with aortal clamping times of $76 \pm 24 \mathrm{~min}$. EEG recording lasted $12.9 \pm 7.2 \mathrm{~h}$ per patient, resulting in $1267 \mathrm{~h}$ of EEG data. Five patients remained intubated and were mechanically ventilated at the end of the EEG recording. One of them received $30 \mathrm{mg} / \mathrm{h}$ propofol. Delirium, as defined by the CAM-ICU, was observed in 9 patients on day 1 (9\%) and 9 patients on day 4 (9\%). In 5 patients, the delirium existed at both times.

$33 \%$ of the patients showed abnormal EEG patterns such as periodic discharges, rhythmic delta activity, rhythmic or sporadic spikes, sharp-and-slow-waves and spikes-and-waves (Table 1). In 9 of these patients (9\% overall), the EEG pattern showed evolution in amplitude and frequency and was interpreted as having electrographic seizures (Fig. 1). The inter-observer agreement was $86 \%$. None of the patients' seizures were observed by the intensive care staff. The main EEG activity at the beginning of the recording time was suppressed or showed a burst-suppression pattern, presumably due to analgo-sedative medication, whereas at the end of EEG recording, the main EEG activity in all patients was an alpha or theta rhythm.

Secondary analysis revealed that the prevalence of electrographic seizures was associated with the incidence of postoperative delirium on the first postoperative day (relative risk 8.09; 95\% confidence interval [2.63; 24.84]; $p_{X 2}<0.01$; effect size $\left.\mathrm{w}=0.388\right)$ and in patients with prolonged delirium at days 1 and 4 (relative risk 6.74; 95\% confidence interval $\left.[1.29,35.2] ; p_{X 2}=0.06\right)$. Patients with electrographic seizures were older (78 vs. 69 years; $p=0.03$ ) and female ( $6 \mathrm{f}$ vs. $3 \mathrm{~m} ; p=0.01$ ) and tended to achieve lower results in pre-surgical MMSE, though this did not reach statistical significance $(26.9 \pm 3.5$ vs. $28.5 \pm 1.4 ; p=0.08)$. The duration of cardiopulmonary bypass, lowest temperature, BIS level and blood pressure due to bypass were not different in patients with abnormal EEG patterns or electrographic seizures. The used dosages of neither clorazepate nor tranexamic acid varied in the observed groups. Four patients had suffered

\section{Table 1 Abnormal EEG findings of the individual patients}

\begin{tabular}{ll}
\hline Electrographic seizures & $\mathbf{9 \%}(\mathbf{9 / 1 0 0 )}$ \\
\hline Generalized rhythmic delta activity (GRDA) & $14 \%(14 / 100)$ \\
\hline Plus superimposed sharp waves/spikes (+S) & $5 \%(5 / 100)$ \\
Plus superimposed fast activity (+ F) & $3 \%(3 / 100)$ \\
Generalized rhythmic spike and wave (GSW) & $2 \%(2 / 100)$ \\
Bilateral independent periodic discharges (BIPD) & $2 \%(2 / 100)$ \\
Plus superimposed rhythmic activity (+R) & $1 \%(1 / 100)$ \\
Lateralized rhythmic delta activity (LRA) & $1 \%(1 / 100)$ \\
Lateralized rhythmic spike and wave (LSW) & $3 \%(3 / 100)$ \\
Sporadic epileptiform discharges & $17 \%(17 / 100)$ \\
\hline
\end{tabular}

Electroencephalography (EEG) findings of the patients with abnormal patterns according to $[7,8]$ 

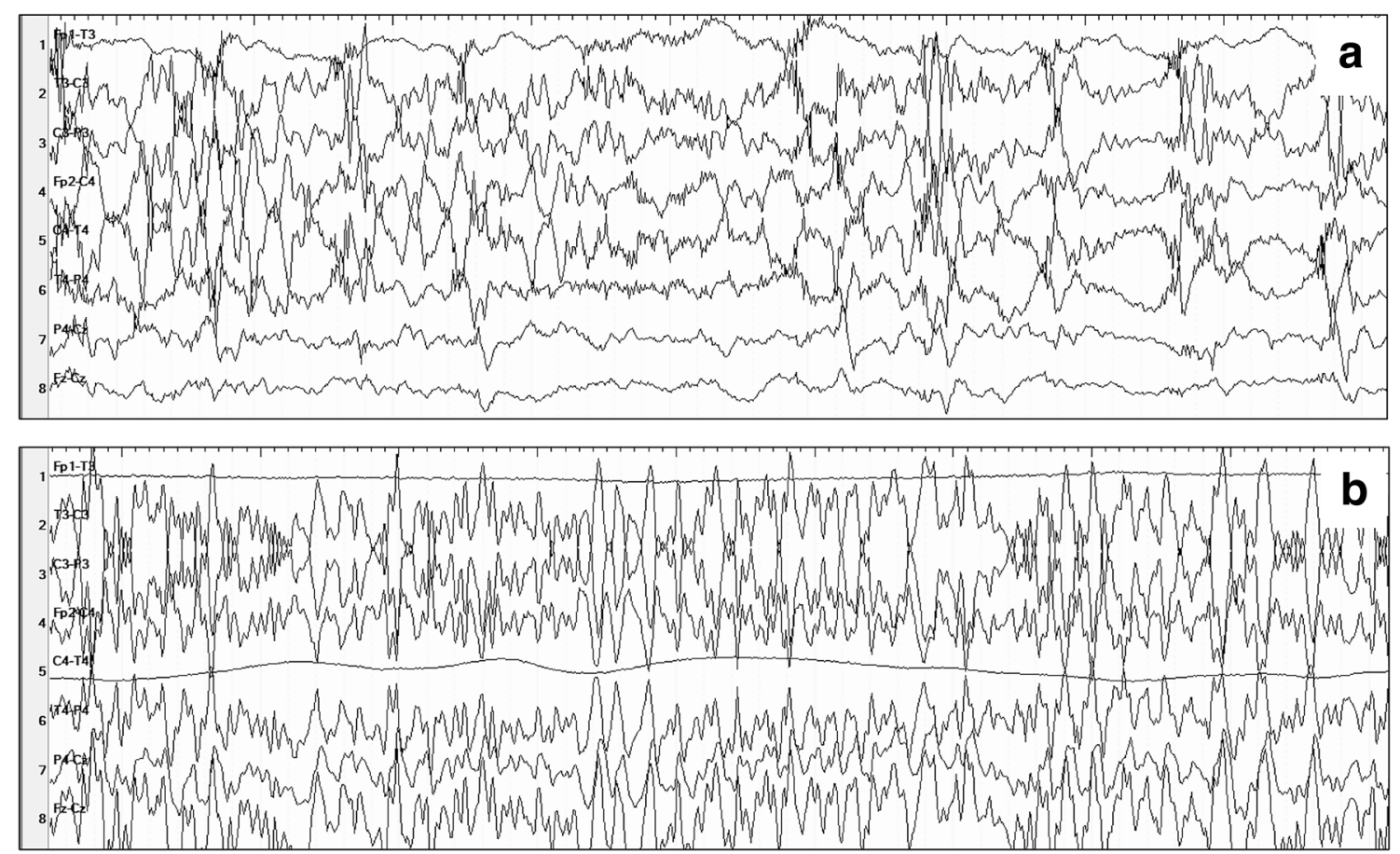

Fig. 1 Exemplary sections of electroencephalograms (EEG) of two patients with electrographic seizures. a Section of left focal rhythmic spike- and poly-spike-wave activity. b Bilateral rhythmic spikes, poly-spikes and poly-spike-wave activity, with artefacts over T3 and T4

a stroke according to their medical history, but this was not associated with an abnormal EEG pattern $(p=1.00)$. Patients were not screened for occult ischemic strokes. Five patients-2/33 with and 3/67 without abnormal EEG patterns $(p=1.00)$ - exhibited acute focal neurological symptoms, and ischemic stroke was confirmed by CT or MRI. Delirium compared to no delirium at day 1 was more pronounced in elderly patients (76 vs. 69 years; $p=0.04)$. The comparison between patients with and without electrographic seizures is shown in Table 2. The comparison between patients with abnormal EEG patterns, including electrographic seizures, and without abnormal EEG patterns can be reviewed in Table 3.

\section{Discussion}

This pilot study shows a surprisingly high prevalence of abnormal EEG patterns after open-chamber cardiac surgery. Electrographic seizures were observed in 9/100 (9\%) of patients, and abnormal EEG patterns were observed in 33/100 (33\%) of patients. Electrographic seizures had a significant association with postoperative delirium and with higher age. All electrographic seizures had no obvious clinical correlate and were not observed by ICU staff. They, therefore, could only be detected by comprehensive EEG monitoring.
We present, for the first time, prospectively assessed EEG data in a representative population undergoing elective open-chamber cardiac surgery. The prevalence of electrographic seizures is higher than in previously published studies of adult patients [2-4], since here for the first time a prospective EEG measurement with widely distributed EEG electrodes was applied. In neonatal patients undergoing cardiac surgery, a prevalence of electrographic seizures at $8 \%$ has been reported [9]. Another study investigating EEGs in sepsis patients described a comparable prevalence of non-convulsive seizures at $11 \%$ and periodic discharges at $25 \%$, similar to the findings described here [10]. In principle, sepsis is a systemic inflammatory response to an infectious pathogen, but a systemic inflammatory response also has been observed after cardiopulmonary bypass [11]. Systemic inflammation therefore might be a potential factor leading to abnormal EEG findings and delirium. The current data provide the necessary basis for further investigations regarding the origin and clinical impact of such EEG abnormalities.

This study has some limitations. Patients were not simultaneously monitored by video, and their EEGs were analysed retrospectively. Since there was no continuous observation of the patients, convulsive versus nonconvulsive seizures could not be differentiated. On the 
Table 2 Comparison between patients with and without electrographic seizures

\begin{tabular}{|c|c|c|c|}
\hline & $\begin{array}{l}\text { Patients with electrographic sei- } \\
\text { zures }(n=9)\end{array}$ & $\begin{array}{l}\text { Patients without electrographic sei- } \\
\text { zures }(n=91)\end{array}$ & $p$ value \\
\hline Gender $[\mathrm{f} / \mathrm{m}]$ & $6 / 3$ & $22 / 69$ & 0.01 \\
\hline Age [years] & $78 \pm 2$ & $69 \pm 10$ & 0.03 \\
\hline MMSE & $26.9 \pm 3.5$ & $28.5 \pm 1.4$ & 0.08 \\
\hline Delirium day 1 [n (\%)] & $4(44 \%)$ & $5(6 \%)$ & $<0.01$ \\
\hline Delirium day 4 [n (\%)] & $2(22 \%)$ & $7(8 \%)$ & 0.19 \\
\hline Delirium day $1+4[n(\%)]$ & $2(29 \%)$ & $3(3 \%)$ & 0.06 \\
\hline Preoperative dose of clorazepate [mg] & $16 \pm 14$ & $25 \pm 16$ & 0.10 \\
\hline $\operatorname{AVR/MVR~}[n] /[n]$ & $7 / 2$ & $69 / 20$ & 1.00 \\
\hline Extracorporeal circulation [min] & $114 \pm 53$ & $113 \pm 36$ & 0.44 \\
\hline Aortal clamping time [min] & $74 \pm 42$ & $76 \pm 22$ & 0.15 \\
\hline Deepest temperature $\left[{ }^{\circ} \mathrm{F} /{ }^{\circ} \mathrm{C}\right]$ & $95 \pm 34 / 35 \pm 1$ & $93 \pm 35 / 34 \pm 1$ & 0.53 \\
\hline Time of $\mathrm{BIS}<40$ [min] & $61 \pm 23$ & $62 \pm 44$ & 0.97 \\
\hline Time of MAP < 40 mmHg [min] & $4[2 ; 24]$ & $6[0 ; 63]$ & 0.98 \\
\hline Doses of tranexamic acid [g] & $2.9 \pm 0.5$ & $2.7 \pm 1$ & 0.55 \\
\hline Lengths of ICU stay [days] & $2[1 ; 12]$ & $1[1 ; 26]$ & 0.08 \\
\hline
\end{tabular}

Values given as mean \pm standard deviation, median [min; max] or frequencies $(n)$ and percentage. Statistical analysis was performed with Mann-Whitney $U$ test, Pearson or Fisher Chi-square test

EEG electroencephalogram, $f$ feminine, $m$ masculine, MMSE mini-mental status examination, AVR aortic valve replacement, $M V R$ mitral valve replacement or reconstruction, $B I S$ bispectral index, MAP mean arterial blood pressure, $I C U$ intensive care unit

Table 3 Comparison between patients with and without abnormal EEG pattern

\begin{tabular}{|c|c|c|c|}
\hline & Patients with abnormal EEG $(n=33)$ & $\begin{array}{l}\text { Patients without abnormal EEG } \\
(n=67)\end{array}$ & $p$ values \\
\hline Gender $[\mathrm{f} / \mathrm{m}]$ & $10 / 23$ & $18 / 49$ & 0.72 \\
\hline Age [years] & $71 \pm 9$ & $69 \pm 10$ & 0.17 \\
\hline MMSE & $28 \pm 2$ & $28 \pm 1$ & 0.47 \\
\hline Delirium day 1 [n (\%)] & $5(15 \%)$ & $4(6 \%)$ & 0.15 \\
\hline Delirium day 4 [n (\%)] & $5(15 \%)$ & $4(6 \%)$ & 0.16 \\
\hline Delirium day $1+4[n(\%)]$ & $3(9 \%)$ & $2(3 \%)$ & 0.33 \\
\hline Preoperative dose of clorazepate [mg] & $24 \pm 16$ & $23 \pm 16$ & 0.74 \\
\hline AVR/MVR $[n] /[n]$ & $27 / 5$ & $49 / 17$ & 0.26 \\
\hline Extracorporeal circulation [min] & $114 \pm 48$ & $112 \pm 32$ & 0.43 \\
\hline Aortal clamping time [min] & $76 \pm 31$ & $76 \pm 19$ & 0.35 \\
\hline Deepest temperature $\left[{ }^{\circ} \mathrm{F} /{ }^{\circ} \mathrm{C}\right]$ & $93 \pm 36 / 34 \pm 2$ & $93 \pm 34 / 34 \pm 1$ & 0.67 \\
\hline Time of $\mathrm{BIS}<40$ [min] & $64 \pm 51$ & $61 \pm 39$ & 0.73 \\
\hline Time of MAP < $40 \mathrm{mmHg}$ [min] & $4[0 ; 27]$ & $6[0 ; 63]$ & 0.5 \\
\hline Doses of tranexamic acid [g] & $2.9 \pm 0.5$ & $2.7 \pm 0.8$ & 0.42 \\
\hline Lengths of ICU stay [days] & $1[1 ; 12]$ & $1[1 ; 26]$ & 0.65 \\
\hline
\end{tabular}

Values given as mean \pm standard deviation, median [min; max] or frequencies $(n)$ and percentage. Statistical analysis was performed with Mann-Whitney $U$ test, Pearson or Fisher Chi-square test

EEG electroencephalogram, $f$ feminine, $m$ masculine, MMSE mini-mental status examination, AVR aortic valve replacement, $M V R$ mitral valve replacement or reconstruction, $B I S$ bispectral index, MAP mean arterial blood pressure, ICU intensive care unit

other hand, the intensive care staff did not register any convulsive seizures. Artefacts by passive or active movement of the patients also were not marked in the EEG. The EEG analyser, therefore, widely ignored patterns typical for active or passive movements. The influence of electrographic seizures or abnormal EEG patterns on outcome parameters, on the one hand, and the influence of perioperative procedures on such EEG abnormalities, on the other hand, cannot be calculated, as this study was not powered for this purpose. All described secondary 
calculations in this trial are, therefore, narrative at this point and should be reproduced and systemically evaluated in the context of further studies.

This study was created as a preliminary pilot study to detect the prevalence of EEG abnormalities in the observed population. Therefore, the study was not powered to investigate the relationship between abnormal EEG patterns and secondarily assessed parameters. However, a significant association between electrographic seizures and delirium exists, supporting the hypothesis of a relationship. Delirium occurred in $9 \%$ of patients and was, therefore, considerably less frequent than the $24 \%$ prevalence in the mixed cardiac surgery population described in previous studies [12]. This observation can likely be explained by the fact that delirium assessment in the above-mentioned investigation was carried out at specific times by specially trained staff, whereas in this study, it was done in the context of routine patient care by intensive care and nursing staff who might underestimate the occurrence of hypoactive delirium, which can be harder to identify. Electrographic seizures and delirium were more pronounced in elderly patients. Furthermore, patients presenting electrographic seizures tended to have lower MMSE level prior to surgery, though this did not reach statistical significance. Both age and preexisting cognitive impairment are well-known risk factors for delirium in ICU patients [13]. It should be discussed whether both entities-seizures and deliriummay be independent consequences of major surgery on an aged and potentially already impaired brain. The association between abnormal EEG patterns and delirium in elderly patients should be investigated in further studies, implementing additional assessments for POCD. Assessing POCD is time-consuming and places high demands on personnel resources. To avoid underpowering in POCD studies, a valid power analysis is necessary. Due to the lack of robust prevalence data on seizures in the population of interest, this investigation was performed without postoperative neurocognitive examinations. POCD is defined as a decline in performance in neuropsychological tests relative to preoperative performance. Its frequency strongly depends on the follow-up interval and the applied diagnostic criteria [14]. Cognitive deficits are still measurable in $16-23 \%$ of patients three months after the operation and in $31 \%$ of patients undergoing cardiac bypass surgery even after 3 years [15, 16]. Patients with status epilepticus or repetitive epileptic seizures are known to experience persistent cognitive deficits in memory and learning even one year after the event [17]. Accordingly, the prevalence of delirium and electrographic seizures in this study now enables the performance of a power analysis in further studies; for the investigation of an association between delirium and electrographic seizures, a sample size of 73 patients should be aimed for (alpha 0.05; power 0.8; effect size $w=0.388$ ). In particular, since seizures are potentially treatable with anticonvulsive drugs, the detection and, if necessary, treatment of early postoperative seizures might be of great clinical importance for the incidence of POCD and will be investigated in further trials.

\section{Conclusions}

Postoperative delirium and POCD are relevant complications of open-chamber cardiac surgery. The underlying pathophysiology is largely unknown and most likely multifactorial. The present study reveals for the first time a high incidence of abnormal EEG patterns and clinically silent postoperative electrographic seizures, with an association of the latter to delirium incidence. This finding warrants further studies to determine whether postoperative abnormal EEG activity contributes to prolonged delirium and POCD and, thus, might represent a promising target for perioperative neuroprotection.

\section{Author details \\ ${ }^{1}$ Heart and Brain Research Group, Kerckhoff Heart and Thorax Center, Bad Nauheim, Germany. ${ }^{2}$ Department of Neurology, Justus-Liebig-University, Giessen, Germany. ${ }^{3}$ Department of Neurology, Gesundheitszentrum Wetterau, Bad Nauheim, Germany. ${ }^{4}$ Department of Anaesthesiology, Kerckhoff Heart and Thorax Center, Bad Nauheim, Germany. ${ }^{5}$ Department of Geriatrics, Jung- Stilling Hospital Siegen, Siegen, Germany. ${ }^{6}$ Department of Cardiac Surgery, Kerckhoff Heart and Thorax Center, Bad Nauheim, Germany. ${ }^{7}$ Department of Anaesthesiology, University Medical Center of the Johannes Gutenberg- University, Langenbeckstrasse 1, 55131 Mainz, Germany.}

\section{Acknowledgements}

Open Access funding provided by Projekt DEAL. Data presented in this manuscript are part of a doctoral thesis presented by Fouad Alhaidar.

\section{Author contributions}

The study was designed by PS, TG and MS. Material preparation, data collection and analysis were performed by MT, MJ, PS, FA, MB, JES and MM. EEG analysis was performed by MT and PS. Statistical analyses were performed by MB. The first draft of the manuscript was written by PS, and all authors commented on previous versions of the manuscript. All authors read and approved the final manuscript.

\section{Funding}

The study was unrestrictedly supported by Nihon Kohden Europe GmbH (Rosbach, Germany) by a loan agreement for the EEG monitor.

\section{Compliance with ethical standards}

Conflicts of interest

The authors declare that they have no conflict of interests.

\section{Ethics approval}

Approval of the ethical committee of the Justus Liebig-University Giessen, Germany, approval number 159/14.

Consent to participate

The written informed consent was obtained from all patients. 


\section{Availability of data and materials}

All data generated or analysed during this study are included in this published article and its supplementary information files.

\section{Open Access}

This article is licensed under a Creative Commons Attribution-NonCommercial 4.0 International License, which permits any non-commercial use, sharing, adaptation, distribution and reproduction in any medium or format, as long as you give appropriate credit to the original author(s) and the source, provide a link to the Creative Commons licence, and indicate if changes were made. The images or other third party material in this article are included in the article's Creative Commons licence, unless indicated otherwise in a credit line to the material. If material is not included in the article's Creative Commons licence and your intended use is not permitted by statutory regulation or exceeds the permitted use, you will need to obtain permission directly from the copyright holder.To view a copy of this licence, visit http://creativecommons.org/licen ses/by-nc/4.0/.

\section{Publisher's Note}

Springer Nature remains neutral with regard to jurisdictional claims in published maps and institutional affiliations.

Received: 1 February 2020 Accepted: 27 April 2020

Published online: 13 May 2020

\section{References}

1. Berger M, Terrando N, Smith SK, Browndyke JN, Newman MF, Mathew JP (2018) Neurocognitive function after cardiac surgery: from phenotypes to mechanisms. Anesthesiology 129:829-851

2. Manji RA, Grocott HP, Leake J, Ariano RE, Manji JS, Menkis AH, Jacobsohn E (2012) Seizures following cardiac surgery: the impact of tranexamic acid and other risk factors. Can J Anaesth 59:6-13

3. Goldstone AB, Bronster DJ, Anyanwu AC, Goldstein MA, Filsoufi F, Adams DH, Chikwe J (2011) Predictors and outcomes of seizures after cardiac surgery: a multivariable analysis of 2,578 patients. Ann Thorac Surg 91:514-518

4. Gofton TE, Chu MW, Norton L, Fox SA, Chase L, Murkin JM, Young GB (2014) A prospective observational study of seizures after cardiac surgery using continuous EEG monitoring. Neurocrit Care 21:220-227

5. Young GB, Sharpe MD, Savard M, Al Thenayan E, Norton L, Davies-Schinkel C (2009) Seizure detection with a commercially available bedside EEG monitor and the subhairline montage. Neurocrit Care 11:411-416

6. Tanner AE, Sarkela MO, Virtanen J, Viertio-Oja HE, Sharpe MD, Norton L, Davies-Schinkel C, Young GB (2014) Application of subhairline EEG montage in intensive care unit: comparison with full montage. J Clin Neurophysiol 31:181-186

7. Hirsch LJ, LaRoche SM, Gaspard N, Gerard E, Svoronos A, Herman ST, Mani $\mathrm{R}$, Arif H, Jette N, Minazad Y, Kerrigan JF, Vespa P, Hantus S, Claassen J, Young GB, So E, Kaplan PW, Nuwer MR, Fountain NB, Drislane FW (2013) American clinical neurophysiology society's standardized critical care EEG terminology: 2012 version. J Clin Neurophysiol 30:1-27

8. Noachtar S, Binnie C, Ebersole J, Mauguiere F, Sakamoto A, Westmoreland B (1999) A glossary of terms most commonly used by clinical electroencephalographers and proposal for the report form for the EEG findings. The International Federation of Clinical Neurophysiology. Electroencephalogr Clin Neurophysiol Suppl 52:21-41

9. Naim MY, Gaynor JW, Chen J, Nicolson SC, Fuller S, Spray TL, Dlugos DJ, Clancy RR, Costa LV, Licht DJ, Xiao R, Meldrum H, Abend NS (2015) Subclinical seizures identified by postoperative electroencephalographic monitoring are common after neonatal cardiac surgery. J Thorac Cardiovasc Surg 150:169-178

10. Gilmore EJ, Gaspard N, Choi HA, Cohen E, Burkart KM, Chong DH, Claassen J, Hirsch $L J$ (2015) Acute brain failure in severe sepsis: a prospective study in the medical intensive care unit utilizing continuous EEG monitoring. Intensive Care Med 41:686-694

11. Squiccimarro E, Labriola C, Malvindi PG, Margari V, Guida P, Visicchio G, Kounakis G, Favale A, Dambruoso P, Mastrototaro G, Lorusso R, Paparella D (2019) Prevalence and clinical impact of systemic inflammatory reaction after cardiac surgery. J Cardiothorac Vasc Anesth 33:1682-1690

12. McPherson JA, Wagner CE, Boehm LM, Hall JD, Johnson DC, Miller LR, Burns KM, Thompson JL, Shintani AK, Ely EW, Pandharipande PP (2013) Delirium in the cardiovascular ICU: exploring modifiable risk factors. Crit Care Med 41:405-413

13. Smith M, Meyfroidt G (2017) Critical illness: the brain is always in the line of fire. Intensive Care Med 43:870-873

14. Rudolph JL, Schreiber KA, Culley DJ, McGlinchey RE, Crosby G, Levitsky S, Marcantonio ER (2010) Measurement of post-operative cognitive dysfunction after cardiac surgery: a systematic review. Acta Anaesthesiol Scand 54:663-677

15. Evered L, Scott DA, Silbert B, Maruff P (2011) Postoperative cognitive dysfunction is independent of type of surgery and anesthetic. Anesth Analg 112:1179-1185

16. Knipp SC, Matatko N, Wilhelm H, Schlamann M, Thielmann M, Losch C, Diener HC, Jakob H (2008) Cognitive outcomes three years after coronary artery bypass surgery: relation to diffusion-weighted magnetic resonance imaging. Ann Thorac Surg 85:872-879

17. Power KN, Gramstad A, Gilhus NE, Hufthammer KO, Engelsen BA (2018) Cognitive function after status epilepticus versus after multiple generalized tonic-clonic seizures. Epilepsy Res 140:39-45 